\title{
FREDHOLMNESS AND INDEX OF OPERATORS IN THE WIENER ALGEBRA ARE INDEPENDENT OF THE UNDERLYING SPACE
}

\author{
MARKO LINDNER
}

Abstract. The purpose of this paper is to demonstrate the so-called Fredholm-inverse closedness of the Wiener algebra $\mathscr{W}$ and to deduce independence of the Fredholm property and index of the underlying space. More precisely, we look at operators $A \in \mathscr{W}$ as acting on a family of vector valued $\ell^{p}$ spaces and show that the Fredholm regularizer of $A$ for one of these spaces can always be chosen in $\mathscr{W}$ as well and therefore regularizes $A$ (modulo compact operators) on all of the $\ell^{p}$ spaces under consideration. We conclude that both Fredholmness and the index of $A$ do not depend on the $\ell^{p}$ space that $A$ is considered as acting on.

Mathematics subject classification (2000): 47A53, 47B37, 47L10, 47B36, 46E40.

Key words and phrases: Fredholm operator, Fredholm index, Wiener algebra, operators on $\ell^{p}$ spaces.

\section{REFERENCES}

[1] S. BOCHNER and R. S. PHILLIPS, Absolutely convergent Fourier expansions for non-commutative normed rings, Ann. of Math. 43 (1942), 409-418.

[2] A. BÖTTCHER and B. SilbermAnN, Analysis of Toeplitz Operators, 1st ed. Akademie-Verlag 1989 and Springer 1990; 2nd ed. Springer 2006.

[3] S. N. ChANDLER-WILDE and M. Lindner, Limit Operators, Collective Compactness, and the Spectral Theory of Infinite Matrices, submitted for publication.

[4] J. Diestel, H. JARCHOW and A. TONGE, Absolutely Summing Operators, Cambridge University Press 1995.

[5] I. Gohberg, S. Goldberg and M. A. KAASHOEK, Classes of Linear Operators, Volume I, Birkhäuser 1990.

[6] I. Gohberg and N. KRUPNIK, One-Dimensional Linear Singular Integral Equations, Volume II, Birkhäuser 1992.

[7] W. T. Gowers, A Solution to Banach's Hyperplane Problem, Bulletin of the LMS 26 (1994), no. 6, 523-530.

[8] R. HemPEL and J. Voigt, The spectrum of a Schrödinger operator in $L^{p}$ is p-independent, Commun. Math. Phys. 104 (1986), 243-250.

[9] P. Koszmider, A Banach space of continuous functions with few operators, Math. Ann. 330 (2004), $151-183$.

[10] B. V. LANGE and V. S. RABINOVICH, On the Noether property of multidimensional discrete convolutions, Mat. Zametki 37 (1985), no. 3, 407-421 (Russian).

[11] M. LINDNER: Infinite Matrices and their Finite Sections: An Introduction to the Limit Operator Method, Frontiers in Mathematics, Birkhäuser 2006.

[12] M. LINDNER: Fredholmness and index of operators in the Wiener algebra are independent of the underlying space, Preprint Nr. 2008-01, TU Chemnitz (2008).

[13] A. PIETSCH and J. WeNZEL, Orthonormal Systems and Banach Space Geometry, Cambridge University Press 1998.

[14] G. PlebaneK, A construction of a Banach space $C(K)$ with few operators, Topology and its Applications 143 (2004), 217-239.

$[15]$ V. S. RABINOVICH and S. RoCH, The Fredholm index of locally compact band-dominated operators on $L^{p}(\mathbb{R})$, Integral Equations and Operator Theory, 57 (2007), no. 2, 263-281. 
[16] V. S. RabinOvich, S. Roch and J. RoE, Fredholm indices of band-dominated operators, Integral Equations Operator Theory 49 (2004), no. 2, 221-238.

[17] V. S. Rabinovich, S. Roch and B. Silbermann, Fredholm Theory and Finite Section Method for Band-dominated operators, Integral Equations Operator Theory 30 (1998), no. 4, 452-495.

[18] V. S. Rabinovich, S. Roch and B. Silbermann, Limit Operators and Their Applications in Operator Theory, Birkhäuser 2004.

[19] S. Roch, Band-dominated operators on $\ell^{p}$ spaces: Fredholm indices and finite sections, Acta Sci. Math. 70 (2004), no. 3-4, 783-797.

[20] I. M. SigAL, A generalized Weyl theorem and $L^{p}$ spectra of Schrödinger operators, J. Oper. Theory 13 (1985), 119-129.

[21] B. SIMON, Brownian motion, $L^{p}$ properties of Schrödinger operators and the localization of binding, J. Funct. Anal. 35 (1980), 215-225.

[22] I. JA. SNEIBERG, The solvability of linear equations in interpolational families of Banach spaces, (Russian), Dokl. Akad. Nauk SSSR 212 (1973), 57-59. 